\title{
Right Ventricular Dysfunction in Patients with Acute STEMI, Radionuclide-Based Assessment
}

\author{
Samir El-Hadidy', Abdalla Mohammed ${ }^{1}$, Shaaban Alramlawy², Waheed Radwan', \\ Mohamed Affify ${ }^{1}$ \\ ${ }^{1}$ Critical Care Medicine Department, Cairo, Egypt \\ ${ }^{2}$ Biophysics Department, Faculty of Medicine, Cairo University, Cairo, Egypt \\ Email: samirelhadidy81@yahoo.com
}

How to cite this paper: El-Hadidy, S., Mohammed, A., Alramlawy, S., Radwan, W. and Affify, M. (2019) Right Ventricular Dysfunction in Patients with Acute STEMI, Radionuclide-Based Assessment. World Journal of Cardiovascular Diseases, 9, 915-929. https://doi.org/10.4236/wjcd.2019.912081

Received: October 1, 2019

Accepted: December 17, 2019

Published: December 20, 2019

Copyright $\odot 2019$ by author(s) and Scientific Research Publishing Inc. This work is licensed under the Creative Commons Attribution International License (CC BY 4.0).

http://creativecommons.org/licenses/by/4.0/

\begin{abstract}
Background: An acute myocardial infarction, particularly one that is large and transmural, can produce alterations in the topography of both the infarcted and noninfarcted regions of the ventricle. Cardiac remodeling after acute myocardial infarction (AMI) is characterized by molecular and cellular mechanisms involving both left and right ventricles. Objective: To study the right ventricular affection in patients with acute myocardial infarction of the left ventricle and treated with primary PCI. Methods: The study was done in the critical care department, Cairo University (in which 2,500 primary percutaneous coronary interventions (PCIs) are done annually). Forty patients with acute ST-elevation myocardial infarction (STEMI) were subjected to primary (PCI), we excluded all patients with RV infarction and patients with pulmonary hypertension. RV function was assessed by first pass radionuclide angiocardiography within 48 hours of admission. Results: Regarding the incidence of right ventricular dysfunction in patients with acute left ventricular STEMI who treated with 1ry PCI, there were 21 patients (52.5\%) with RV $\mathrm{EF} \%<40 \%, 16$ patients $(40 \%)$ with RV peak emptying rate (PER) $<1.9$ $\mathrm{EDV} / \mathrm{s}, 18$ patients (45\%) with RV peak filing rate (PFR) $<2.5 \mathrm{EDV} / \mathrm{s}$ and 19 patients $(47.5 \%)$ with RV time to peak filling rate (TPFR) $>180 \mathrm{msec}$. There was a significant relationship between right ventricular dysfunction and duration of ICU stay, impairment of LV systolic function, failure of complete resolution of ST segment elevation, failure of early peaking of cardiac enzymes, occurrence of in-hospital complications and one year mortality. Conclusion: The incidence of right ventricular dysfunction in patients with acute left ventricular STEMI is higher than expected and has a negative impact on their outcome.
\end{abstract}

\section{Keywords}

STEMI, RV Affection, First Pass, Radionuclide 


\section{Introduction}

The LV and RV are working as a "functional syncytium" and the two ventricles cannot be dissociated in an independent manner.

Knowledge about the role of the right ventricle in health and disease historically has lagged behind that of the left ventricle.

More precise characterization of right ventricular function should improve our understanding of pathophysiology and permit more rational management of cardiac disorders.

An acute myocardial infarction, particularly one that is large and transmural, can produce alterations in the topography of both the infarcted and noninfarcted regions of the ventricle [1].

The mechanisms leading to RV dysfunction following AMI involving the LV are not completely clear, but it is frequently assumed that LV failure causes pulmonary hypertension ( $\mathrm{PH}$ ) and increased RV afterload leading to RV remodeling and dysfunction. In addition, infarction or ischemia of the RV and/or the septum is common in patients with AMI and can also contribute to abnormal RV systolic function, and also neurohormonal activation or inflammation may be involved in subtle right ventricular affection in patients with acute MI.

$\mathrm{RV}$ remodeling and dysfunction following AMI are a part of a global biventricular remodeling response due to altered wall stress which is further aggravated by neurohormonal activation during and after AMI. Cardiac remodeling after acute myocardial infarction (AMI) is characterized by molecular and cellular mechanisms involving both left and right ventricles, and biventricular failure identifies patients with an extremely unfavorable prognosis [1].

Data on the association between right ventricular (RV) function and adverse events after acute myocardial infarction (AMI) are scarce and the association between right ventricular function and other clinical outcomes after MI was less clear.

Post-infarction cardiac remodeling leading to HF represents a "dangerous intersection" in AMI patients, and biventricular failure is considered to be the terminal stage of cardiac remodeling.

It was found that impaired right ventricular function is an adverse prognostic factor in patients with inferior infarction, particularly in those with impaired left ventricular function.

Evaluation of RV structure and function in patients with cardiopulmonary disorders became a vital step in the management. Many tools are available for right ventricular assessment beside the clinical examination and electrocardiogram (ECG); echocardiography gives a good evaluation of RV structure and function [2]. Radionuclide-based techniques provide reliable and geometrically independent assessments of RVEF. Cardiac magnetic resonance imaging (MRI) is increasingly used as a tool in the evaluation of right ventricle [3] [4]. 


\section{Methods}

The study was conducted on forty patients admitted to the critical care department, Cairo University with acute STEMI and subjected to primary PCI. Our study was done within 48 hours of admission.

\section{Inclusion criteria:}

All patients who experienced acute ST-segment elevation myocardial infarction (STEMI) and subjected to primary percutaneous coronary intervention (PCI) of the infarct-related artery (IRA) were included in the study within 48 hours of admission.

Exclusion criteria:

- Patients with a coexisting RV infarction who were diagnosed by 12 leads ECG with recorded right-sided leads.

- Patients received thrombolytic therapy.

- Atrial fibrillation.

- Pulmonary hypertension, $\mathrm{PH}$ was defined as an estimated PASP $\geq 35 \mathrm{mmHg}$ [5].

- A pacer wire in the right ventricle.

- Patients who had been diagnosed with non-ischemic dilated cardiomyopathy before the onset of MI.

The following data were obtained from all patients and included in the current work: Personal data (name, age and gender), Medical history, date, cause of admission and chest pain duration before admission.

Routine laboratory tests and Serial cardiac enzymes (creatine kinase, the CK-MB isoenzyme, troponin $\mathrm{T}$ and lactate dehydrogenase enzyme) and we noticed the early peaking of cardiac enzymes if there was an abrupt initial increase of creatine kinase levels, more than two-fold over the upper normal or baseline elevated values at two hours of reperfusion therapy.

Twelve leads ECG was obtained with right sided leads were recorded if inferior MI was present. We document the rhythm and the site of MI.

Right ventricular infarction is suggested by the presence of:

- ST elevation in V1, ST elevation in lead III > lead II.

- ST elevation in V1 > V2.

- ST elevation in V1 + ST depression in V2.

Right ventricular infarction is confirmed by the presence of ST elevation in the right-sided leads (V3R-V6R).

The reduction of sum of ST segment elevation after primary PCI was documented, and graded it in 3 categories:

- $\geq 50 \%$ reduction of sum of ST segment elevation.

- $20 \%-50 \%$ reduction of sum of ST segment elevation.

- $<20 \%$ reduction of sum of ST segment elevation.

Radionuclide-based techniques:

Assessments of the left and right ventricular function by technetium-99 m first pass radionuclide angiocardiography. 
We analyzed the initial transit of intravenously injected technetium- $99 \mathrm{~m}$ bolus as it traverses the central circulation by the gamma camera and assessed biventricular systolic function, ventricular volume measurements and ventricular compliance through peak filling and emptying rates.

A large proximal vein in the right ante-cubital fossa was cannulated with a large gauge cannula, Electrocardiography (ECG) gated list mode acquisition was done]. Acquisition was started a few seconds before the injection. Simultaneous biplanar acquisition with a double-headed camera has been done. The acquisition duration was about 30 seconds, in order to allow for complete transition through the central circulation.

Finally, we observe the course of the patient as regard occurrence of in-hospital complications, duration of ICU stay and the mortality (in-hospital, 28-days and one year mortality).

Statistical analysis of the data, data were coded and entered using the statistical package SPSS (Statistical Package for the Social Sciences) version 24. Data were summarized using mean, standard deviation, median, minimum and maximum in quantitative data and using frequency (count) and relative frequency (percentage) for categorical data. Comparisons between quantitative variables were done using the non-parametric Mann-Whitney test [6]. For comparing categorical data, Chi-square $\left(\chi^{2}\right)$ test was performed. Exact test was used instead when the expected frequency is less than 5 [7]. P-values less than 0.05 were considered as statistically significant.

\section{Results}

Our study included forty patients ( 32 men and 8 women) aged $54 \pm 10$ years admitted to ICU with acute STEMI and treated with primary PCI (Table 1 \& Table 2), of whom 17 patients (42.5\%) were diabetic, 13 patients (57.5\%) were hypertensive, 32 patients (80\%) were smokers, 23 patients (57.5\%) had dyslipidemia, 8 patients (20\%) had past history of ischemic heart disease and 16 patients $(40 \%)$ had family history of ischemic heart disease (Table 3 ). The mean duration of chest pain before admission to the ICU was $4.43 \pm 1.89$ hours. The mean Door to Balloon duration was $2.1 \pm 0.53$ hours. The mean duration of ICU stay in our study was $6.45 \pm 2.53$ days (Table 4). During hospitalization, 14 patients (35\%) developed in-hospital complications (cardiogenic shock, arrhythmias and recurrent ischemia) while the rest of patients had a smooth course (Table 5 and Figure 1). Three patients developed cardiogenic shock, five patients suffered from recurrent ischemia and six patients developed arrhythmias (4 patients developed atrial fibrillation, 1 patient developed non-sustained ventricular tachycardia and 1 patient developed sustained ventricular tachycardia) (Table 6).

Cardiogenic shock was defined in our study based on the following established clinical criteria: 1) Hypotension-systolic blood pressure $<90 \mathrm{mmHg}$ for more than $30 \mathrm{~min}$ or the need for vasopressor/mechanical support to achieve this blood pressure; 2) evidence of end-organ hypoperfusion with at least one of 
the following: altered mental status, cold clammy skin or extremities, urine out-put less than $30 \mathrm{ml} / \mathrm{h}$, or elevated serum lactate greater than $2 \mathrm{mmol} / \mathrm{L}$ [8]. Recurrent ischemia defined as the persistence of chest pain due to myocardial ischemia after revascularization.

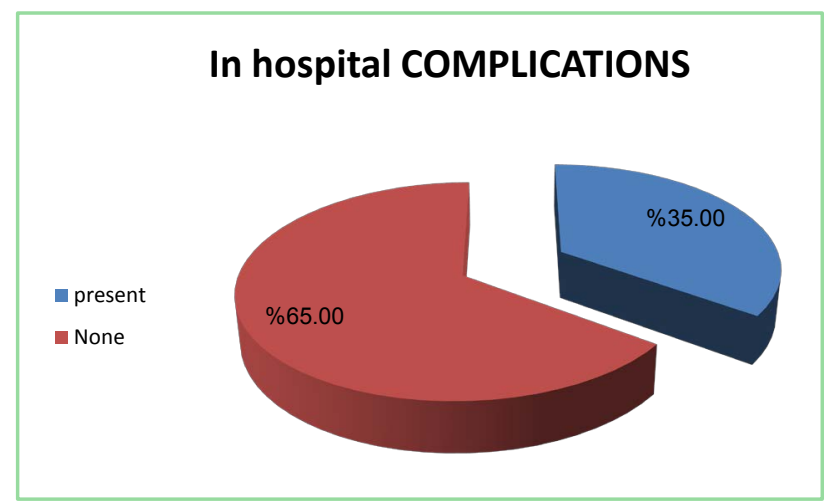

Figure 1. Incidence of in-hospital complications. Three patients developed cardiogenic shock, five patients suffered from recurrent ischemia and six patients developed arrhythmias (4 patients developed atrial fibrillation, 1 patient developed non-sustained ventricular tachycardia and 1 patient developed sustained ventricular tachycardia).

Table 1. Demographic characteristics-Age.

\begin{tabular}{cccccc}
\hline & Mean & Standard Deviation & Median & Minimum & Maximum \\
\hline Age & 54.02 & 10.19 & 53.50 & 33.00 & 76.00 \\
\hline
\end{tabular}

Table 2. Demographic characteristics-Gender.

\begin{tabular}{cccc}
\hline & & Count & $\%$ \\
\hline \multirow{2}{*}{ Gender } & Male & 32 & $80.0 \%$ \\
& Female & 8 & $20.0 \%$ \\
\hline
\end{tabular}

Table 3. Clinical characteristics of the participants.

\begin{tabular}{cccc}
\hline & & Count & $\%$ \\
\hline \multirow{2}{*}{ DM } & DM & 17 & $42.5 \%$ \\
& Not diabetic & 23 & $57.5 \%$ \\
HTN & Hypertensive & 13 & $32.5 \%$ \\
& Normotensive & 27 & $67.5 \%$ \\
Smoking & Smoker & 32 & $80.0 \%$ \\
& Not smoker & 8 & $20.0 \%$ \\
Dyslipidemia & Dyslipidemia & 23 & $57.5 \%$ \\
& Normal & 17 & $42.5 \%$ \\
IHD (Previous ACS) & Positive history & 8 & $20.0 \%$ \\
& No history & 32 & $80.0 \%$ \\
Family history & Positive history & 16 & $40.0 \%$ \\
& No history & 24 & $60.0 \%$ \\
\hline
\end{tabular}


Table 4. Duration of ICU stay.

\begin{tabular}{lccccc}
\hline & Mean & Standard Deviation & Median & Minimum & Maximum \\
\hline ICU Stay (days) & 6.45 & 2.53 & 6.00 & 3.00 & 18.00 \\
\hline
\end{tabular}

Table 5. Incidence of in-hospital complications.

\begin{tabular}{lccc}
\hline & & Count & $\%$ \\
\hline \multirow{2}{*}{ (In hospital COMPLICATIONS) } & Present & 14 & $35.0 \%$ \\
& None & 26 & $65.0 \%$ \\
\hline
\end{tabular}

Table 6. The developed in-hospital complications.

\begin{tabular}{cccc}
\hline & & Count & $\%$ \\
\hline \multirow{2}{*}{ Cardiogenic shock } & Shocked & 3 & $7.5 \%$ \\
Recurrent ischemia & Non shocked & 37 & $92.5 \%$ \\
& Patients did not develop ischemia & 35 & $12.5 \%$ \\
Arrhythmias & Patients developed arrhythmias & 6 & $87.5 \%$ \\
& Patients did not develop arrhythmias & 34 & $15 \%$ \\
\hline
\end{tabular}

Coronary angiographic data:

Infarct related artery (IRA) was left anterior descending artery (LAD) in 30 patients $(75 \%)$, right coronary artery (RCA) in 8 patients $(20 \%)$ and left circumflex artery (LCX) in only $2(5 \%)$ patients. There was one lesion in 16 patients (40\%), two lesions in 17 patients (42.5\%) and three lesions in 7 patients (17.5\%). We found that TIMI flow was 0 (pre PCI) in all patients, II (post PCI) in 9 patients (22.5\%) and III (post PCI) in 31patients (77.5\%) (Table 7).

No documented in-hospital and 28-days mortality while in one year, 2 mortalities were identified (Table 8).

First pass radionuclide angiocardiography results revealed that the mean LV EF\% was $40.44 \% \pm 8.75 \%$ and the LV peak emptying rate (PER) was $2.28 \pm 0.64$ $\mathrm{EDV} / \mathrm{s}$, the mean LV peak filling rate (PFR) was $2.84 \pm 1.03 \mathrm{EDV} / \mathrm{s}$, the time to peak filling rate (TPFR) was $180 \pm 50 \mathrm{msec}$., the mean RV EF\% was $36.68 \% \pm$ $8.43 \%$ and the RV peak emptying rate (PER) was $2.28 \pm 0.62 \mathrm{EDV} / \mathrm{s}$, the mean $\mathrm{RV}$ peak filling rate (PFR) was $2.39 \pm 1.05 \mathrm{EDV} / \mathrm{s}$ and the time to peak filling rate (TPFR) was $179.32 \pm 48 \mathrm{msec}$ (Table 9 \& Figure 2 \& Figure 3).

On studying the incidence of right ventricular dysfunction among these patients, we found that there were 21 patients (52.5\%) with RV EF\% $<40 \%, 16$ patients $(40 \%)$ with RV peak emptying rate (PER) $<1.9 \mathrm{EDV} / \mathrm{s}, 18$ patients $(45 \%)$ with RV peak filing rate (PFR) $<2.5 \mathrm{EDV} / \mathrm{s}$ and 19 patients (47.5\%) with RV time to peak filling rate (TPFR) $>180 \mathrm{msec}$ (Table 10 \& Figure 4).

On studying the relation between the site of $\mathrm{MI}$ and the right ventricular affection, there was no statistically significant difference. Also on studying the re- 
lation between the Infarct related artery (IRA) and the right ventricular dysfunction, there was no statistically significant difference (Table 11).

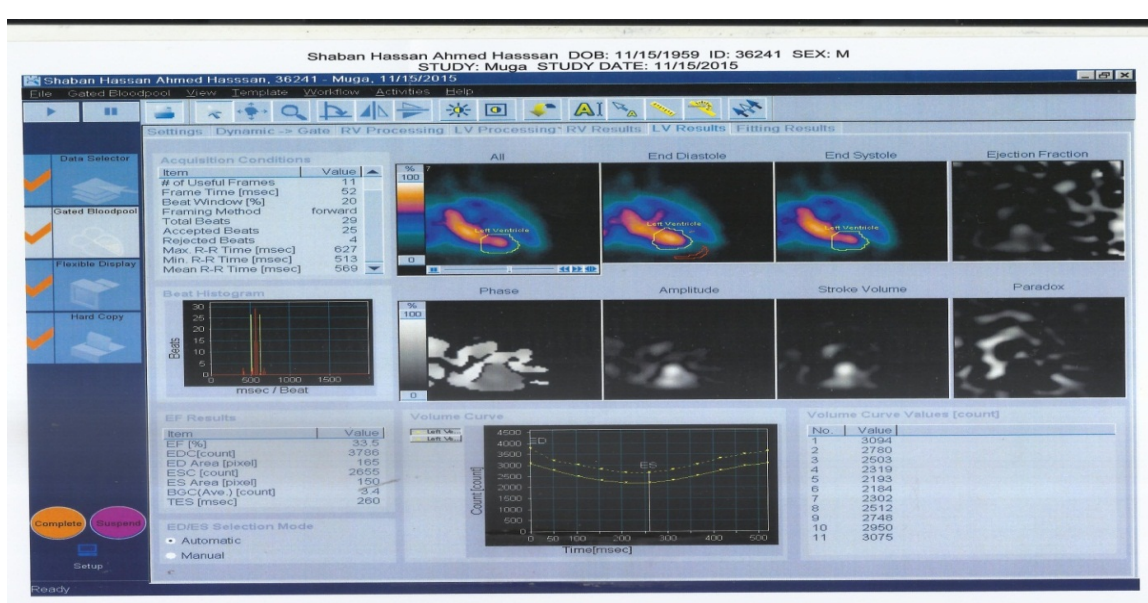

Figure 2. First pass radionuclide angiocardiography: Time activity curve.

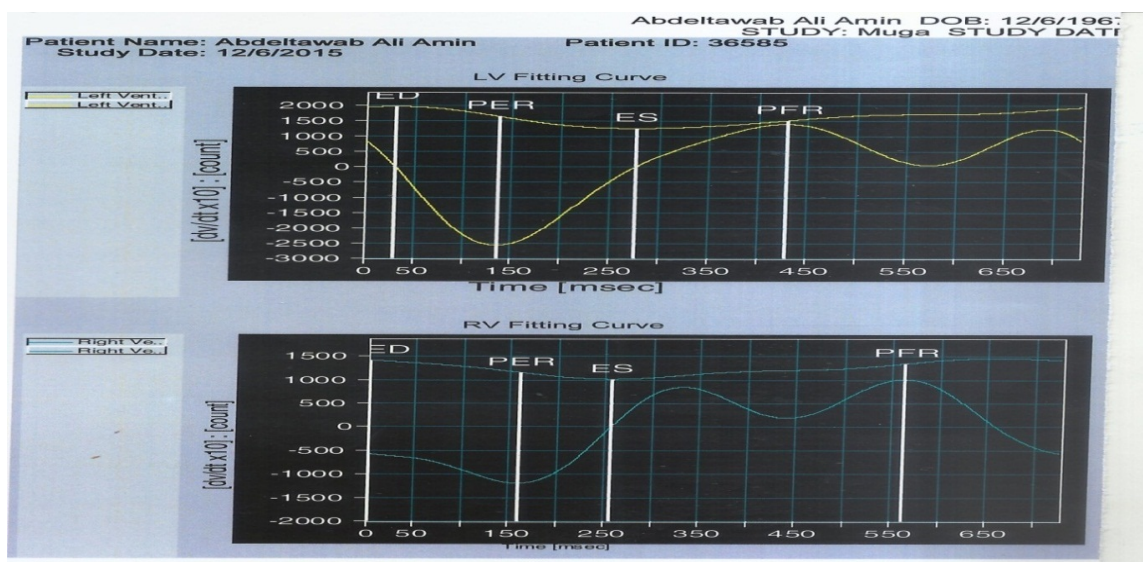

Figure 3. First pass radionuclide angiocardiography: LV \& RV fitting curves.

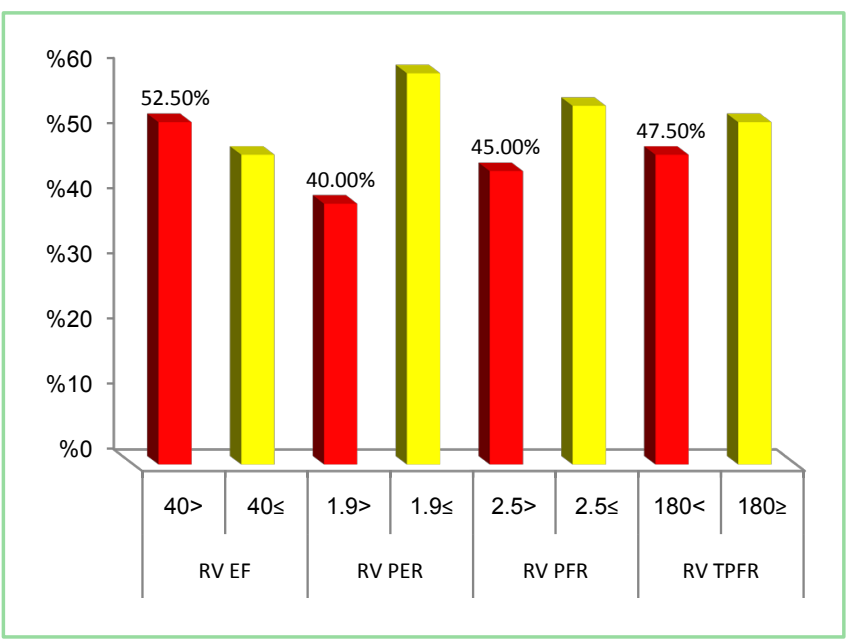

Figure 4. Incidence of right ventricular dysfunction in patients with acute STEMI after 1ry PCI. EF: Ejection fraction, PER: Peak emptying rate, PFR: Peak filling rate, RV: Right ventricle, TPFR: Time to peak filling rate. 
Table 7. Coronary angiographic data.

\begin{tabular}{cccc}
\hline & & Count & $\%$ \\
\hline \multirow{2}{*}{ IRA } & (LAD) & 30 & $75 \%$ \\
& (LCX) & 2 & $5.0 \%$ \\
Number of Significant & (RCA) & 8 & $20.0 \%$ \\
lesions on angiography & One significant lesion & 16 & $40.0 \%$ \\
& Two significant lesions & 17 & $42.5 \%$ \\
Post PCI TIMI flow grade & Three significant lesions & 7 & $17.5 \%$ \\
& TIMI flow II & 9 & $22.5 \%$ \\
& TIMI flow III & 31 & $77.5 \%$ \\
\hline
\end{tabular}

LAD: Left anterior descending artery, LCX: Left circumflex artery, RCA: Right coronary artery.

Table 8. The mortality.

\begin{tabular}{cccc}
\hline & & Count & $\%$ \\
\hline \multirow{2}{*}{ In-hospital Mortality } & Non-survivors & 0 & $0.0 \%$ \\
& survivors & 40 & $100.0 \%$ \\
\multirow{2}{*}{ 28 days Mortality } & Non-survivors & 0 & $0.0 \%$ \\
& survivors & 40 & $100.0 \%$ \\
One year mortality & Non-survivors & 2 & $5.0 \%$ \\
& survivors & 38 & $95.0 \%$ \\
\hline
\end{tabular}

Table 9. First pass radionuclide angiocardiography results.

\begin{tabular}{cccccc}
\hline & Mean & Standard Deviation & Median & Minimum & Maximum \\
\hline LV EF [\%] & 40.44 & 8.75 & 41.00 & 20.00 & 60.00 \\
LV PER (EDV/s) & 2.28 & .64 & 1.95 & 1.50 & 3.60 \\
LV PFR (EDV/s) & 2.84 & 1.03 & 2.55 & 1.60 & 4.80 \\
LV TPFR [msec] & 180.08 & 50.05 & 183.00 & 106.00 & 362.00 \\
RV EF [\%] & 36.68 & 8.43 & 37.00 & 20.40 & 51.00 \\
RV PER (EDV/s) & 2.28 & .62 & 2.00 & 1.50 & 3.70 \\
RV PFR (EDV/s) & 2.93 & 1.05 & 2.65 & 1.50 & 5.00 \\
RV TPFR [msec] & 179.32 & 49.08 & 177.00 & 105.00 & 328.00 \\
\hline
\end{tabular}

EF: Ejection fraction, PER: Peak emptying rate, PFR: Peak filling rate, RV: Right ventricle, TPFR: Time to peak filling rate.

Table 10. Percentage of Right ventricular affection in patients with acute STEMI after 1ry PCI (by first pass radionuclide angiography).

\begin{tabular}{cccc}
\hline & & Count & $\%$ \\
\hline \multirow{2}{*}{ RV EF } & $<40$ & 21 & $52.5 \%$ \\
& $\geq 40$ & 19 & $47.5 \%$ \\
RV PER & $<1.9$ & 16 & $40.0 \%$ \\
& $\geq 1.9$ & 24 & $60.0 \%$ \\
RV PFR & $<2.5$ & 18 & $45.0 \%$ \\
& $\geq 2.5$ & 22 & $55.0 \%$ \\
RV TPFR & $>180$ & 19 & $47.5 \%$ \\
& $\leq 180$ & 21 & $52.5 \%$ \\
\hline
\end{tabular}

EF: Ejection fraction, PER: Peak emptying rate, PFR: Peak filling rate, RV: Right ventricle, TPFR: Time to peak filling rate. 
Table 11. Right ventricular affection and Infarct related artery (IRA).

\begin{tabular}{|c|c|c|c|c|c|c|c|c|}
\hline & & \multicolumn{6}{|c|}{ IRA } & \multirow{3}{*}{ P-value } \\
\hline & & \multicolumn{2}{|c|}{ LAD } & \multicolumn{2}{|c|}{ LCX } & \multicolumn{2}{|c|}{ RCA } & \\
\hline & & Count & $\%$ & Count & $\%$ & Count & $\%$ & \\
\hline \multirow{2}{*}{ RV EF } & $<40$ & 18 & $60.0 \%$ & 0 & $0.0 \%$ & 3 & $37.5 \%$ & \multirow{2}{*}{0.219} \\
\hline & $\geq 40$ & 12 & $40.0 \%$ & 2 & $100.0 \%$ & 5 & $62.5 \%$ & \\
\hline \multirow{2}{*}{ RV PER } & $<1.9$ & 15 & $50.0 \%$ & 0 & $0.0 \%$ & 1 & $12.5 \%$ & \multirow{2}{*}{0.090} \\
\hline & $\geq 1.9$ & 15 & $50.0 \%$ & 2 & $100.0 \%$ & 7 & $87.5 \%$ & \\
\hline \multirow{2}{*}{ RV PFR } & $<2.5$ & 17 & $56.7 \%$ & 0 & $0.0 \%$ & 1 & $12.5 \%$ & \multirow{2}{*}{0.060} \\
\hline & $\geq 2.5$ & 13 & $43.3 \%$ & 2 & $100.0 \%$ & 7 & $87.5 \%$ & \\
\hline \multirow{2}{*}{ RV TPFR } & $>180$ & 17 & $56.7 \%$ & 0 & $0.0 \%$ & 2 & $25.0 \%$ & \multirow{2}{*}{0.109} \\
\hline & $\leq 180$ & 13 & $43.3 \%$ & 2 & $100.0 \%$ & 6 & $75.0 \%$ & \\
\hline
\end{tabular}

EF: Ejection fraction, PER: Peak emptying rate, PFR: Peak filling rate, RV: Right ventricle, TPFR: Time to peak filling rate.

There was a statistically significant relation between Rt ventricular dysfunction and the duration of ICU stay, we observed that patients with right ventricular dysfunction had a prolonged ICU stay, P-values are 0.008, 0.009, 0.003 and 0.016 (Table 12 \& Figure 5).

It was found that right ventricular dysfunction in our patients was related to the impairment of LV systolic function, and the relation was statistically significant $(\mathrm{P}<0.001)$ (Table 13).

We found that the right ventricle was more affected with post PCI TIMI flow II than post PCI TIMI flow III as observed from RV EF, PER and PFR (P-value = $0.021,0.018$ and 0.006 respectively) (Table 14 ).

We noticed also that the right ventricle was more affected with failure of resolution of ST segment after the primary PCI $(\mathrm{P}<0.05)$, and with failure of occurrence of early peaking of cardiac enzymes $(\mathrm{P} \leq 0.001)$ (Table $15 \&$ Table 16).

There was a statistically significant relation between RT ventricular dysfunction and in-hospital complications (P-values are 0.002, 0.003, 0.001 and 0.004) (Table 17 \& Figure 6). There was a relation between right ventricular affection and the one year mortality but it did not reach the statistically significant value, $\mathrm{P} \geq 0.05$ (Table 18 \& Figure 7).

\section{Discussion}

The right ventricle (RV) has historically received less attention than its counterpart of the left side of the heart, yet there is a substantial body of evidence showing that RV size and function are perhaps equally important in predicting adverse outcomes in cardiovascular diseases [9] [10] [11].

Ventricular function can be assessed with radionuclide techniques by two methods, first-pass radionuclide angiocardiography and equilibrium radionuclide angiocardiography (ERNA) [12]. Both techniques have the advantage of being independent of geometric assumption and have been validated extensively. 


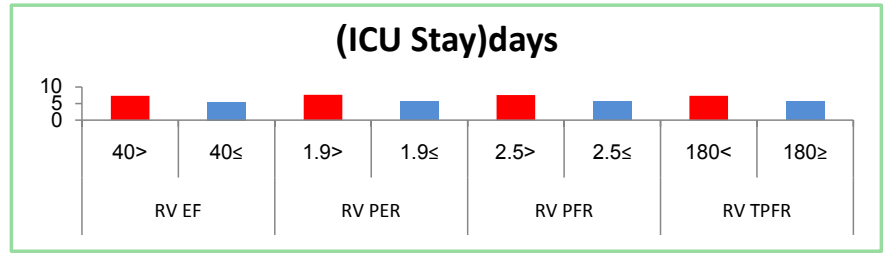

Figure 5. Right ventricular affection and duration of ICU stay. EF: Ejection fraction, PER: Peak emptying rate, PFR: Peak filling rate, RV: Right ventricle, TPFR: Time to peak filling rate.

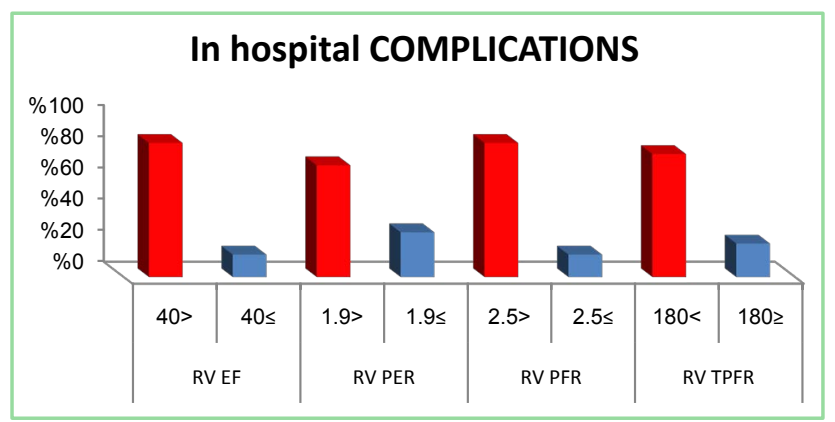

Figure 6. Relation of right ventricular affection and occurrence of in-hospital complications. EF: Ejection fraction, PER: Peak emptying rate, PFR: Peak filling rate, RV: Right ventricle, TPFR: Time to peak filling rate.

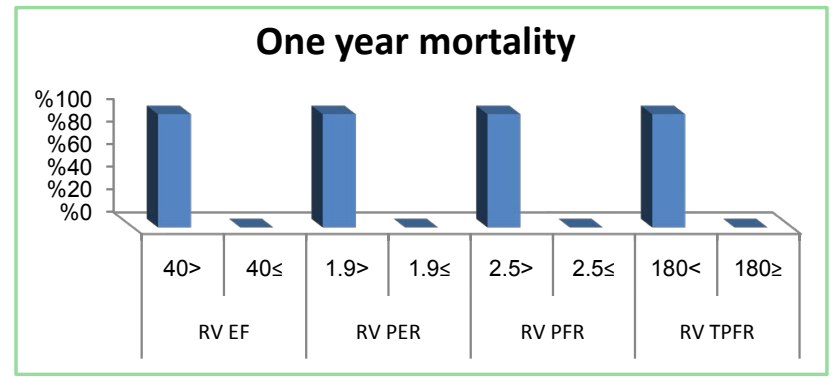

Figure 7. Right ventricular affection and one year mortality. EF: Ejection fraction, PER: Peak emptying rate, PFR: Peak filling rate, RV: Right ventricle, TPFR: Time to peak filling rate.

Table 12. Right ventricular affection and duration of ICU stay.

\begin{tabular}{|c|c|c|c|c|c|c|c|}
\hline & & \multicolumn{5}{|c|}{ ICU Stay (days) } & \multirow[b]{2}{*}{ P-value } \\
\hline & & Mean & SD & Median & Minimum & Maximum & \\
\hline \multirow{2}{*}{ RV EF } & $<40$ & 7.33 & 2.92 & 7.00 & 3.00 & 18.00 & \multirow{2}{*}{0.008} \\
\hline & $\geq 40$ & 5.47 & 1.58 & 5.00 & 3.00 & 9.00 & \\
\hline \multirow{2}{*}{ RV PER } & $<1.9$ & 7.62 & 3.20 & 7.00 & 3.00 & 18.00 & \multirow{2}{*}{0.009} \\
\hline & $\geq 1.9$ & 5.67 & 1.61 & 5.00 & 3.00 & 9.00 & \\
\hline \multirow{2}{*}{ RV PFR } & $<2.5$ & 7.61 & 3.05 & 7.00 & 3.00 & 18.00 & \multirow{2}{*}{0.003} \\
\hline & $\geq 2.5$ & 5.50 & 1.50 & 5.00 & 3.00 & 9.00 & \\
\hline \multirow{2}{*}{ RV TPFR } & $>180$ & 7.37 & 3.00 & 7.00 & 3.00 & 18.00 & \multirow{2}{*}{0.016} \\
\hline & $\leq 180$ & 5.62 & 1.69 & 5.00 & 3.00 & 9.00 & \\
\hline
\end{tabular}

EF: Ejection fraction, PER: Peak emptying rate, PFR: Peak filling rate, RV: Right ventricle, TPFR: Time to peak filling rate. 
Table 13. Right ventricular affection and impairment of LV systolic function (EF\%).

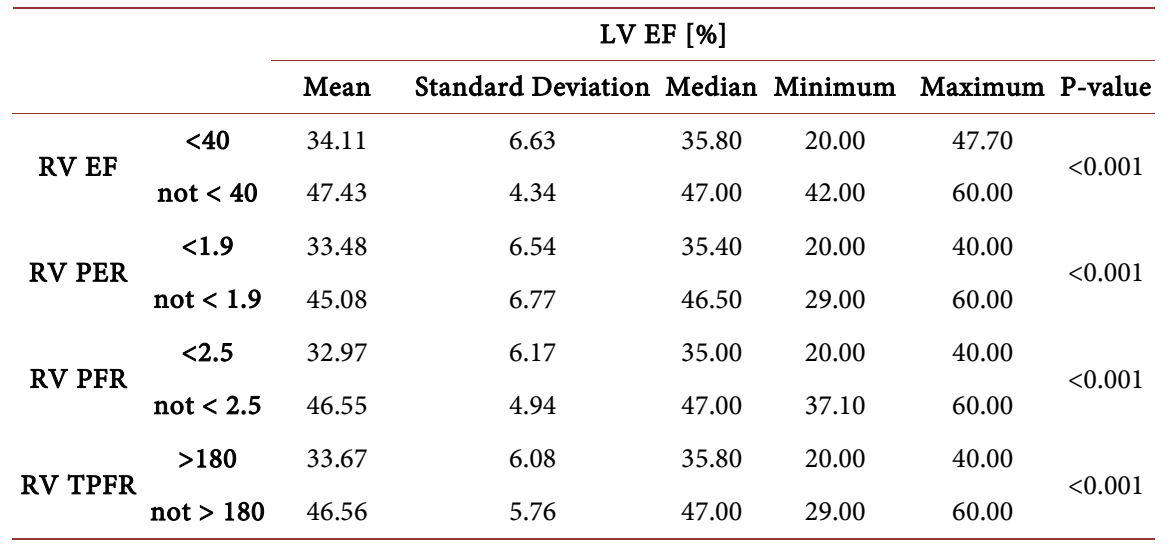

EF: Ejection fraction, PER: Peak emptying rate, PFR: Peak filling rate, RV: Right ventricle, TPFR: Time to peak filling rate.

Table 14. Right ventricular and TIMI flow (post PCI).

\begin{tabular}{|c|c|c|c|c|c|c|}
\hline & & \multicolumn{4}{|c|}{ Post PCI TIMI flow grade } & \multirow{3}{*}{ P-value } \\
\hline & & \multicolumn{2}{|c|}{ TIMI flow II } & \multicolumn{2}{|c|}{ TIMI flow III } & \\
\hline & & Count & $\%$ & Count & $\%$ & \\
\hline \multirow{2}{*}{ RV EF } & $<40$ & 8 & $88.9 \%$ & 13 & $41.9 \%$ & \multirow{2}{*}{0.021} \\
\hline & $\geq 40$ & 1 & $11.1 \%$ & 18 & $58.1 \%$ & \\
\hline \multirow{2}{*}{ RV PER } & $<1.9$ & 7 & $77.8 \%$ & 9 & $29.0 \%$ & \multirow{2}{*}{0.018} \\
\hline & $\geq 1.9$ & 2 & $22.2 \%$ & 22 & $71.0 \%$ & \\
\hline \multirow{2}{*}{ RV PFR } & $<2.5$ & 8 & $88.9 \%$ & 10 & $32.3 \%$ & \multirow{2}{*}{0.006} \\
\hline & $\geq 2.5$ & 1 & $11.1 \%$ & 21 & $67.7 \%$ & \\
\hline \multirow{2}{*}{ RV TPFR } & $>180$ & 7 & $77.8 \%$ & 12 & $38.7 \%$ & \multirow{2}{*}{0.060} \\
\hline & $\leq 180$ & 2 & $22.2 \%$ & 19 & $61.3 \%$ & \\
\hline
\end{tabular}

EF: Ejection fraction, PER: Peak emptying rate, PFR: Peak filling rate, RV: Right ventricle, TPFR: Time to peak filling rate.

Table 15. Right ventricular affection and ST segment elevation resolution after the primary PCI.

\begin{tabular}{|c|c|c|c|c|c|c|c|c|}
\hline & & \multicolumn{6}{|c|}{ ST elevation resolution (ECG) } & \multirow{3}{*}{ P-value } \\
\hline & & \multicolumn{2}{|c|}{$<20 \%$} & \multicolumn{2}{|c|}{$20 \%-50 \%$} & \multicolumn{2}{|c|}{$>50 \%$} & \\
\hline & & Count & $\%$ & Count & $\%$ & Count & $\%$ & \\
\hline \multirow{2}{*}{ RV EF } & $<40$ & 6 & $100.0 \%$ & 13 & $81.2 \%$ & 2 & $11.1 \%$ & \multirow{2}{*}{$<0.001$} \\
\hline & $\geq 40$ & 0 & $0.0 \%$ & 3 & $18.8 \%$ & 16 & $88.9 \%$ & \\
\hline \multirow{2}{*}{ RV PER } & $<1.9$ & 4 & $66.7 \%$ & 10 & $62.5 \%$ & 2 & $11.1 \%$ & \multirow{2}{*}{0.002} \\
\hline & $\geq 1.9$ & 2 & $33.3 \%$ & 6 & $37.5 \%$ & 16 & $88.9 \%$ & \\
\hline \multirow{2}{*}{ RV PFR } & $<2.5$ & 6 & $100.0 \%$ & 10 & $62.5 \%$ & 2 & $11.1 \%$ & \multirow{2}{*}{$<0.001$} \\
\hline & $\geq 2.5$ & 0 & $0.0 \%$ & 6 & $37.5 \%$ & 16 & $88.9 \%$ & \\
\hline \multirow{2}{*}{ RV TPFR } & $>180$ & 6 & $100.0 \%$ & 11 & $68.8 \%$ & 2 & $11.1 \%$ & \multirow{2}{*}{$<0.001$} \\
\hline & $\leq 180$ & 0 & $0.0 \%$ & 5 & $31.2 \%$ & 16 & $88.9 \%$ & \\
\hline
\end{tabular}

EF: Ejection fraction, PER: Peak emptying rate, PFR: Peak filling rate, RV: Right ventricle, TPFR: Time to peak filling rate. 
Table 16. Right ventricular affection and Early peaking of cardiac enzymes after primary PCI.

\begin{tabular}{|c|c|c|c|c|c|c|}
\hline & & \multicolumn{4}{|c|}{ Eary peaking after $\mathrm{PCI}$} & \multirow{3}{*}{ P-value } \\
\hline & & \multicolumn{2}{|c|}{ Yes } & \multicolumn{2}{|c|}{ No } & \\
\hline & & Count & $\%$ & Count & $\%$ & \\
\hline \multirow{2}{*}{ RV EF } & $<40$ & 9 & $32.1 \%$ & 12 & $100.0 \%$ & \multirow{2}{*}{$<0.001$} \\
\hline & $\geq 40$ & 19 & $67.9 \%$ & 0 & $0.0 \%$ & \\
\hline \multirow{2}{*}{ RV PER } & $<1.9$ & 5 & $17.9 \%$ & 11 & $91.7 \%$ & \multirow{2}{*}{$<0.001$} \\
\hline & $\geq 1.9$ & 23 & $82.1 \%$ & 1 & $8.3 \%$ & \\
\hline \multirow{2}{*}{ RV PFR } & $<2.5$ & 6 & $21.4 \%$ & 12 & $100.0 \%$ & \multirow{2}{*}{$<0.001$} \\
\hline & $\geq 2.5$ & 22 & $78.6 \%$ & 0 & $0.0 \%$ & \\
\hline \multirow{2}{*}{ RV TPFR } & $>180$ & 8 & $28.6 \%$ & 11 & $91.7 \%$ & \multirow{2}{*}{$<0.001$} \\
\hline & $\leq 180$ & 20 & $71.4 \%$ & 1 & $8.3 \%$ & \\
\hline
\end{tabular}

EF: Ejection fraction, PER: Peak emptying rate, PFR: Peak filling rate, RV: Right ventricle, TPFR: Time to peak filling rate.

Table 17. Relation of right ventricular affection and in-hospital complications.

\begin{tabular}{|c|c|c|c|c|c|c|}
\hline & & \multicolumn{4}{|c|}{ (In hospital COMPLICATIONS) } & \multirow{3}{*}{ P-value } \\
\hline & & \multicolumn{2}{|c|}{ Yes } & \multicolumn{2}{|c|}{ No } & \\
\hline & & Count & $\%$ & Count & $\%$ & \\
\hline \multirow{2}{*}{ RV EF } & $<40$ & 12 & $85.7 \%$ & 9 & $34.6 \%$ & \multirow{2}{*}{0.002} \\
\hline & $\geq 40$ & 2 & $14.3 \%$ & 17 & $65.4 \%$ & \\
\hline \multirow{2}{*}{ RV PER } & $<1.9$ & 10 & $71.4 \%$ & 6 & $23.1 \%$ & \multirow{2}{*}{0.003} \\
\hline & $\geq 1.9$ & 4 & $28.6 \%$ & 20 & $76.9 \%$ & \\
\hline \multirow{2}{*}{ RV PFR } & $<2.5$ & 12 & $85.7 \%$ & 6 & $23.1 \%$ & \multirow{2}{*}{$<0.001$} \\
\hline & $\geq 2.5$ & 2 & $14.3 \%$ & 20 & $76.9 \%$ & \\
\hline \multirow{2}{*}{ RV TPFR } & $>180$ & 11 & $78.6 \%$ & 8 & $30.8 \%$ & \multirow{2}{*}{0.004} \\
\hline & $\leq 180$ & 3 & $21.4 \%$ & 18 & $69.2 \%$ & \\
\hline
\end{tabular}

EF: Ejection fraction, PER: Peak emptying rate, PFR: Peak filling rate, RV: Right ventricle, TPFR: Time to peak filling rate.

Table 18. Right ventricular affection and one year mortality.

\begin{tabular}{|c|c|c|c|c|c|c|}
\hline & & \multicolumn{4}{|c|}{ One year mortality } & \multirow{3}{*}{ P-value } \\
\hline & & \multicolumn{2}{|c|}{ alive } & \multicolumn{2}{|c|}{ dead } & \\
\hline & & Count & $\%$ & Count & $\%$ & \\
\hline \multirow{2}{*}{ RV EF } & $<40$ & 19 & $50.0 \%$ & 2 & $100.0 \%$ & \multirow{2}{*}{0.488} \\
\hline & $\geq 40$ & 19 & $50.0 \%$ & 0 & $0.0 \%$ & \\
\hline \multirow{2}{*}{ RV PER } & $<1.9$ & 14 & $36.8 \%$ & 2 & $100.0 \%$ & \multirow{2}{*}{0.154} \\
\hline & $\geq 1.9$ & 24 & $63.2 \%$ & 0 & $0.0 \%$ & \\
\hline \multirow{2}{*}{ RV PFR } & $<2.5$ & 16 & $42.1 \%$ & 2 & $100.0 \%$ & \multirow{2}{*}{0.196} \\
\hline & $\geq 2.5$ & 22 & $57.9 \%$ & 0 & $0.0 \%$ & \\
\hline \multirow{2}{*}{ RV TPFR } & $>180$ & 17 & $44.7 \%$ & 2 & $100.0 \%$ & \multirow{2}{*}{0.219} \\
\hline & $\leq 180$ & 21 & $55.3 \%$ & 0 & $0.0 \%$ & \\
\hline
\end{tabular}

EF: Ejection fraction, PER: Peak emptying rate, PFR: Peak filling rate, RV: Right ventricle, TPFR: Time to peak filling rate. 
The first-pass technique has the disadvantage of having a lower count density, technical difficulties, whereas the equilibrium technique has difficulty separating the right atrium from the right ventricle and has no benefits for the long-term follow up and the clinical application due to the large dosage and long collection duration [13]. There are some obvious advantages with first pass study as the acquisition of data in less than 30 seconds; determination of RV function with less overlap of activity in other chambers; the use of multiple radiopharmaceuticals and the ability to obtain high-quality studies in even morbidly obese individuals [14].

In our study, on determining the incidence of right ventricular dysfunction among the patients with acute MI after 1ry PCI, we found that there were 21 (52.5\%) patients with RV EF\% < 40\%, 16 (40\%) patients with RV peak emptying rate $(\mathrm{PER})<1.9 \mathrm{EDV} / \mathrm{s}, 18(45 \%)$ patients with $\mathrm{RV}$ peak filing rate (PFR) $<2.5$ EDV/s and 19 (47.5\%) patients with RV time to peak filling rate (TPFR) $>180$ msec. In the study done by Polak J. F. et al. [15], the predictive value of radionuclide ventriculography was studied in 34 patients with atherosclerotic coronary artery disease, when a right ventricular ejection fraction of less than $35 \%$ was used as a discriminant, there were 21 patients (62\%) with a depressed right ventricular ejection fraction. In the study done by Jensen C. J. et al. in year 2010 [16], Fifty patients with first-ST-segment elevation MI underwent 1.5-T cardiac MRI immediately after successful percutaneous coronary intervention. Right ventricular involvement was diagnosed with cardiac MRI in 27 patients (54\%).

In our study, there was a significant statistical relation between RT ventricular dysfunction and the duration of ICU stay, we observed that patients with right ventricular dysfunction had a prolonged ICU stay, P-values are 0.008, 0.009, 0.003 and 0.016 for radionuclide parameters. In disagreement with our data is the study of Jensen C. J. et al. in year 2010 [16] in which they found that duration of ICU treatment did not differ significantly between the group with and that without $\mathrm{RV}$ involvement $(3 \pm 0.9,2.7 \pm 0.9$ respectively $\mathrm{P} 0.3)$, in spite of it was longer with the group of RV involvement, it did not reach a statistical significance.

We found that the right ventricle was more affected with post PCI TIMI flow II than post PCI TIMI flow III as observed from RV EF, PER and PFR (P: 0.021, 0.018 and 0.006 respectively). We noticed also that the right ventricle was more affected with failure of resolution of ST segment after the primary PCI $(\mathrm{P}<$ 0.05). Also, we found that the right ventricle was more affected with failure of occurrence of early peaking of cardiac enzymes $(\mathrm{P} \leq 0.001)$, we did not find similar studies to compare our results with it.

Our results revealed a significant statistical relation between RT ventricular affection and in-hospital complications (P-values are 0.002, 0.003, 0.001 and $0.004)$. There was a relation between right ventricular affection and the one year mortality but it did not reach the statistically significant value, $\mathrm{P} \geq 0.05$. This may be explained by the small number of died patients, which may be related to that the mean LV ejection fraction in our study was $40.4 \%$ and most of patients 
(26 patients) had a smooth course during the study. Shah PK and his colleagues in year 1986 [17] performed a study on 114 consecutive patients with acute myocardial infarction (AMI), radionuclide ventriculography was done to determine the left and right ventricular ejection fractions (EFs). They found that among patients with an acute myocardial infarction, the presence of a low radionuclide right ventricular ejection fraction $(<0.38)$ plus low left ventricular ejection fraction $(<0.30)$ results in 3 times the 1 -year mortality of patients with poor left ventricular function alone.

\section{Conclusion}

The incidence of right ventricular dysfunction in patients with acute left ventricular STEMI is higher than expected and has a negative impact on their outcome.

\section{Conflicts of Interest}

The authors declare no conflicts of interest regarding the publication of this paper.

\section{References}

[1] John, J. and Ryan, J. (2015) Diagnosing and Treating the Failing Right Heart. Current Opinion in Cardiology, 30, 292-299. https://doi.org/10.1097/HCO.0000000000000164

[2] Leonardo, A., Zornoff, M., Skali, H., Marc, A., feffer, P., Martin, S., et al. (2002) Right Ventricular Dysfunction and Risk of Heart Failure and Mortality after Myocardial Infarction. Journal of the American College of Cardiology, 39, 1450-1455. https://doi.org/10.1016/S0735-1097(02)01804-1

[3] Kumar, A., Abdel-Aty, H., Kriedemann, I., et al. (2006) Contrast-Enhanced Cardiovascular Magnetic Resonance Imaging of Right Ventricular Infarction. Journal of the American College of Cardiology, 48, 1969-1976. https://doi.org/10.1016/j.jacc.2006.05.078

[4] Bleeker, G., Steendijk, P., Holman, E., Yu, C., Breithardt, O., Kaandorp, T., et al. (2006) Assessing Right Ventricular Function: The Role of Echocardiography and Complementary Technologies. Heart, 92, 19-26. https://doi.org/10.1136/hrt.2005.082503

[5] McQuillan, B., Picard, M., Leavitt, M. and Weyman, A. (2001) Clinical Correlates and Reference Intervals for Pulmonary Artery Systolic Pressure among Echocardiographically Normal Subjects. Circulation, 104, 2797-2802. https://doi.org/10.1161/hc4801.100076

[6] Chan, Y., et al. (2003) Biostatistics 102: Quantitative Data-Parametric \& Non-Parametric Tests. Singapore Medical Journal, 44, 391-396.

[7] Chan, Y., et al. (2003) Biostatistics 103: Qualitative Data-Tests of Independence. Singapore Medical Journal, 44, 498-503.

[8] Thiele, H., Ohman, E.M., Desch, S., Eitel, I. and de Waha, S. (2015) Management of Cardiogenic Shock. European Heart Journal, 36, 1223-1230. https://doi.org/10.1093/eurheartj/ehv051

[9] Antoni, M., Scherptong, R.W., Atary, J.Z., Boersma, E., et al. (2009) Prognostic 
Value of Right Ventricular Function in Patients after Acute Myocardial Infarction Treated with Primary Percutaneous Coronary Intervention. Circulation, 914, 366.

[10] Kjaergaard, J., Petersen, C., Kjaer, A., Schaadt, B., Oh, J. and Hassager, C. (2006) Evaluation of Right Ventricular Volume and Function by 2D and 3D Echocardiography Compared to MRI. European Journal of Echocardiography, 7, 430-438. https://doi.org/10.1016/j.euje.2005.10.009

[11] Saito, K., et al. (2009) Comprehensive Evaluation of Left Ventricular Strain Using Speckle Tracking Echocardiography in Normal Adults: Comparison of Three Dimensional and Two Dimensional Approaches. American Society of Echocardiography, 22, 1025-1030. https://doi.org/10.1016/j.echo.2009.05.021

[12] Corbett, J., Akinboboye, O., Bacharach, S., Borer, J., Botvinick, E., DePuey, G., et al. (2006) Equilibrium Radionuclide Angiocardiography. Journal of Nuclear Cardiolo$g y$, 13, 56-79. https://doi.org/10.1016/j.nuclcard.2006.08.007

[13] Jain, D. and Zaret, B. (1992) Assessment of Right Ventricular Function: Role of Nuclear Imaging Techniques. Cardiology Clinics, 10, 23-39. https://doi.org/10.1016/S0733-8651(18)30253-4

[14] Schad, N. and Nickel, O. (1980) Assessment of Ventricular Function with First-Pass Angiocardiography. Cardiovascular Radiology, 2, 149-160. https://doi.org/10.1007/BF02552060

[15] Polak, J., Holman, L., Wynne, J. and Colucci, W. (1983) Right Ventricular Ejection Fraction: An Indicator of Increased Mortality in Patients with Congestive Heart Failure Associated with Coronary Artery Disease. Journal of the American College of Cardiology, 2, 217-224. https://doi.org/10.1016/S0735-1097(83)80156-9

[16] Jensen, C., Jochims, M., Hunold, P., Sabin, G., Schlosser, T. and Bruder, O. (2010) Right Ventricular Involvement in Acute Left Ventricular Myocardial Infarction: Prognostic Implications of MRI Findings. American Journal of Roentgenology, 194, 592-598. https://doi.org/10.2214/AJR.09.2829

[17] Shah, P., Maddahi, J., Staniloff, H., Ellrodt, A., Pichler, M., Swan, H. and Berman, D. (1986) Variable Spectrum and Prognostic Implications of Left and Right Ventricular Ejection Fractions in Patients with and without Clinical Heart Failure after Acute Myocardial Infarction. American Journal of Cardiology, 58, 387-393.

https://doi.org/10.1016/0002-9149(86)90001-9 